\title{
Analysis of the Parameter Space of a Metric for Registering 3D Vascular Images
}

\author{
Stephen R. Aylward, Sue Weeks, and Elizabeth Bullitt \\ Department of Radiology \\ Computer-Aided Diagnosis and Display Lab \\ The University of North Carolina at Chapel Hill
}

\begin{abstract}
We present a new metric for registering 3D images of vasculature, and we analyze the rigid-body transformation parameter space of that metric and its derivatives. To quantify and direct a source image's alignment with a target image, this new vascular-image registration system models the vessels in the source image and makes measurements in the target image at a sparse set of transformed points from the centerlines of those models. The system is fast and effective because the measures made at the transformed centerline points incorporate the general geometric properties of tubes and specific model-quality information calculated during the vessel model generation process. Additionally, by adjusting the sample density or scaling the centerline point measures, coarse-tofine registration strategies are directly enabled. We present visualizations of the metric and its derivatives over a range of mis-registrations given different sample densities and different measure scalings using magnetic resonance angiograms, x-ray computed tomography images, and 3D ultrasound images.
\end{abstract}

\section{Introduction and Background}

The registration of $3 \mathrm{D}$ medical images is critical to many clinical tasks. Most computer-based registration research focuses on registering tissue images. Many clinical applications, however, are driven by the patient's vasculature. Vascular imaging modalities include x-ray computed tomography after contrast injection (CTA), magnetic resonance angiography (MRA), and 3D ultrasound (3DUS). Applications that benefit from the registration of vascular images include: quantifying treatment effectiveness for arterio-venous malformations (AVM) as captured by MRA and planning adult-to-adult partial liver transplants using registered CTA to generate digital-subtraction CTA of liver vasculature.

We know of no other vessel-specific 3D image registration metric. If image intensities or vessel centerline features are used to register vascular images, the sparseness and local similarity of these features produce a multitude of local maxima in the transformation parameter space. Others have developed methods for model-based segmentation of coronary arteries (e.g., [1]), but such work assumes one model fits every patient and does not fully exploit ridge criteria 


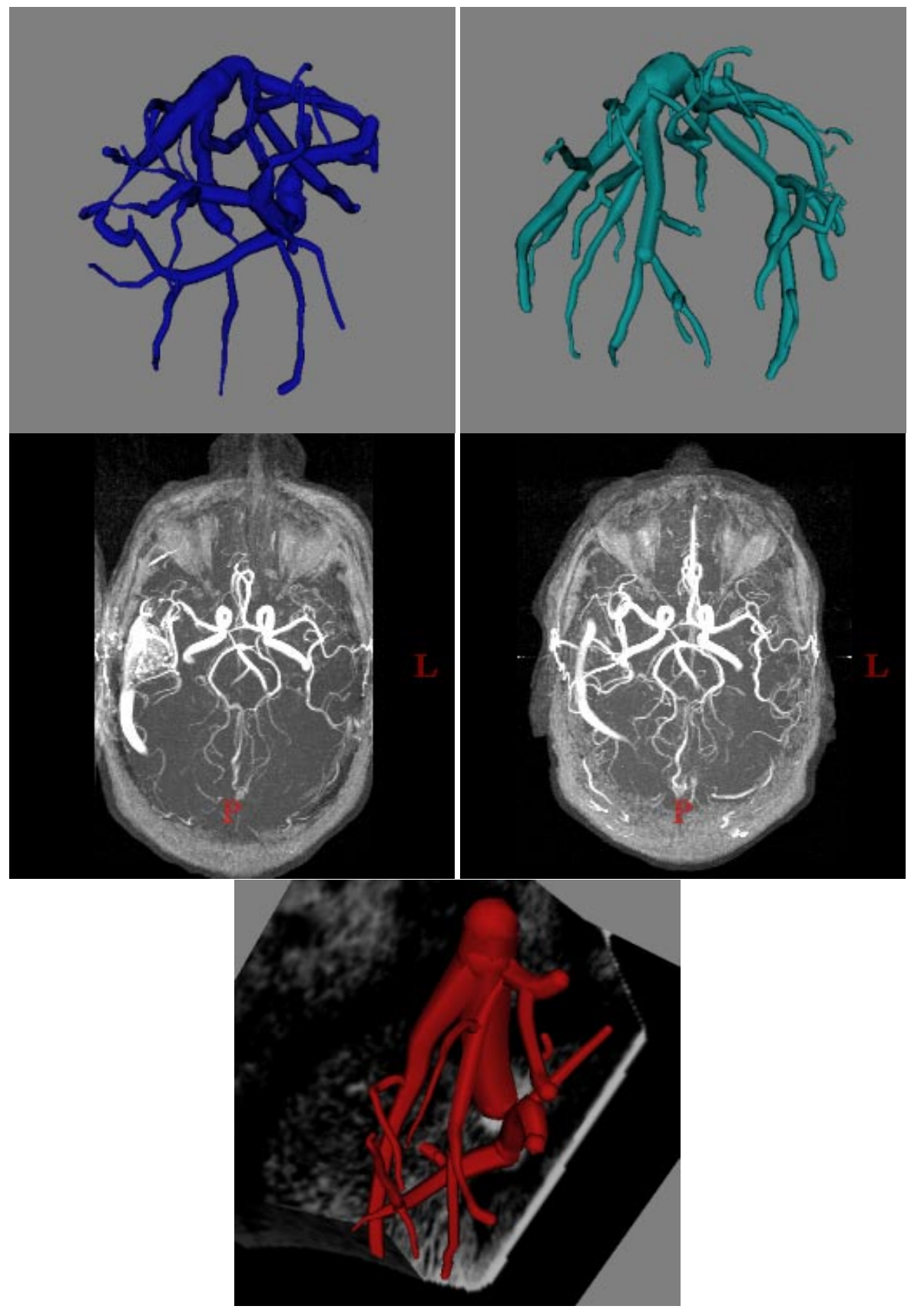

Fig. 1. (Top row) Vessel models from two liver CTA datasets: (left) from data collected during the arterial-phase of contrast and (right) from the venous-phase of contrast. (Middle row) Maximum intensity projections of two MRA datasets (an AVM is visible on the left side of each image): (left) pre-treatment data and (right) three months after radiation therapy treatment. (Bottom row) Vessel models from 3D-US data. 
during registration. We [2] and others $[1,3]$ have previously presented methods for registration of $3 \mathrm{D}$ vascular models with their $2 \mathrm{D}$ projections.

In this paper, we present our $3 \mathrm{D}$ vascular registration metric and illustrate its parameter space using clinical MRA, CTA, and 3D-US data (Fig 1). We show that the metric is maximal when two vascular images are aligned; the metric varies smoothly about that maximum; and for a broad range of transformations, the metric's derivatives point towards the maximum. Additionally, we show that the parameters of this metric control the extent and smoothness of the metric and its parameters about the maximum, thereby enabling coarse-to-fine registration strategies. Furthermore, the metric and its derivatives require minimal computation - even at high sample density, these calculations usually require less than one-half second, so complete $3 \mathrm{D}$ registration can be accomplished in under one minute.

\section{Method and Results}

The registration metric builds on our vessel modeling work [2, 4]. A summary of other vessel modeling methods is given in [5], but none of these other methods provides the information that we have found useful for registration. Specifically, the registration metric requires models of the vessels in the "source image." Our model extraction method produces sub-voxel representations of the centerlines of vessels via a multi-scale ridge traversal process. The centerlines are then used to stabilize a vessel-width estimation process. At each centerline point, therefore, the following information is defined in our vessel models:

$\mathbf{x} \quad$ The position of the centerline point in the source image, $\mathbf{x} \in \Re^{3}$

$r \quad$ The radius of the vessel at $\mathbf{x}$

$\mathbf{I}_{r}(\mathbf{x}) \quad$ The image intensity at $\mathbf{x}$ at scale $r$ (the height ridge of a vessel exists at scales related to the width of the vessel)

$\overrightarrow{\mathbf{v}_{1}}, \overrightarrow{\mathbf{v}_{2}} \quad$ Co-vectors that define the directions normal to the centerline at $\mathbf{x}$

$\lambda_{1}, \lambda_{2} \quad$ The second derivative of the image intensity in directions $\overrightarrow{\mathbf{v}_{1}}$ and $\overrightarrow{\mathbf{v}_{2}}$ ridgeness Quantifies how well a centerline point localizes a ridge. Defined by $\left(\overrightarrow{\mathbf{v}_{1}} \bullet \nabla \mathbf{I}_{r}(\mathbf{x})\right)^{2}+\left(\overrightarrow{\mathbf{v}_{2}} \bullet \nabla \mathbf{I}_{r}(\mathbf{x})\right)^{2}$

ellipticalness Equal to 1 if cross-section at $\mathbf{x}$ is circular. Defined by $\lambda_{2} / \lambda_{1}$ medialness Response from convolving the image at $\mathbf{x}$ with a series of radius $r$ center-on, surround-off kernels oriented along the centerline.

\subsection{Vascular Registration Metric}

The premise of the registration metric is that when two vascular images are aligned, the centerlines from one map to the intensity ridges in the other. This paper considers only rigid-body transforms defined by the constrained matrix $\mathbf{T}$ which we parameterize by its offset vector $\mathbf{o}$ and the Euler rotation parameters $\alpha, \beta$, and $\gamma$. The registration metric, $f(\mathbf{T})$, is a weighted, $w_{i}$, sum of scaled, $\kappa r_{i}$, intensities in the target image, $\mathbf{I}$, at a sub-sampling, $n$, of the transformed centerline points, $\mathbf{x}_{i} \mathbf{T}$. 


$$
f(\mathbf{T})=\frac{1}{\sum_{i=1}^{n} w_{i}} \sum_{i=1}^{n} w_{i} \mathbf{I}_{\kappa r_{i}}\left(\mathbf{x}_{i} \mathbf{T}\right)
$$

The centerlines are sub-sampled to reduce the number of points at which a volume must be evaluated to quantify the quality of an alignment. This greatly reduces the computation requirements of the metric. Furthermore, we reject any sub-sample point if its ridgeness or its medialness (see Section 2) is less than 0.2 (to eliminate points which are poorly localized). This actually increases the accuracy of the metric when few points are used. The effect of sub-sampling on the shape of metric surface about the metric maximum is illustrated in Fig. 2 On a $500 \mathrm{Mhz}$ Pentium III, for the CT data, to calculate the metric and its gradients requires 0.06 seconds when 46 points ( $\sim$ of every 100 centerline points in the vessel models) are used, 0.39 seconds when 279 points ( $~ 1$ of 20 ) are used, and 1.97 seconds when 1360 points ( $\sim 1$ of $5=\sim 1$ point per voxel a centerline passes through) are used. For the MRA data, instead of evaluating the $10,485,760$ voxels in the data, to calculate the metric and its gradients requires 0.02 seconds for 76 points, 0.09 seconds for 513 points, and 0.49 seconds for 2559 points. The MRA data requires less time per evaluation since the typical centerline point exists at a smaller scale in the MRA data compared to the CTA data. A coarse-to-fine registration strategy can by implimented by changing the density of the sub-sampling during transformation parameter optimization.
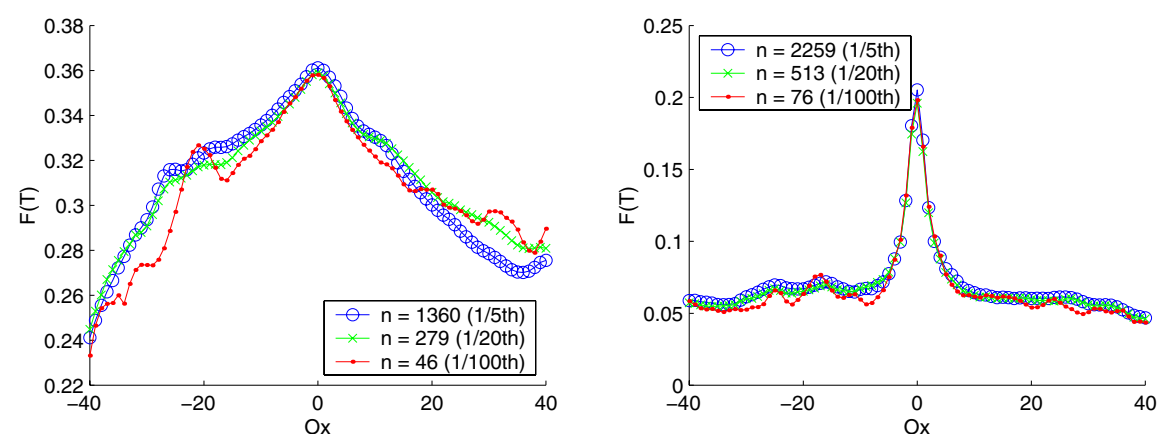

Fig. 2. Metric value for model translations of \pm 40 voxels $(\sim \pm 5 \mathrm{~cm})$ in the $x$-axis direction for the CTA (left) and the MRA (right) data given different sub-samplings. For CTA, vessel models from arterial-phase data were registered with venous-phase data. For MRA, vessel models from pre-treatment data were registered with postradiation treatment data. For MRA and CTA, the registered models defined the 0offset transformations (the location of the metric maximum). These datasets contained non-rigid deformations including missing and extra vessels, but the metric is insensitive.

Since the spatial extent of small vessels is limited, they are more drastically affected by image noise, and they are less informative given large-scale misregistrations. We therefore weight vessels based on their radius (Equ. 2). This 
weighting could be balanced during transformation optimization to impliment a coarse-to-fine registration strategy.

$$
w_{i}=\frac{2}{1+e^{-2 r_{i}}}
$$

Via the scalings $\kappa r_{i}$ in Equ. 1, the measurements made in the target image are made using aperatures proportional to the radius of the vessel expected at that point in the target image. This local blurring creates an intensity ridge, if the point is transformed to a vessel's centerline in the target image. This scaling can be controlled using the metric parameter $\kappa$. If $\kappa$ is increased beyond 1.0, the capture radius of the metric increases, the effect of noise is decreased, and larger vessels are given additional emphasis in determining the qualify of the registration. By starting with $\kappa$ large, and decreasing $\kappa$ to 1.0 as a registration process iterates, a coarse-to-fine registration strategy is effected (Fig. 3). Note the multiple local maxima at $\kappa=0.0$.
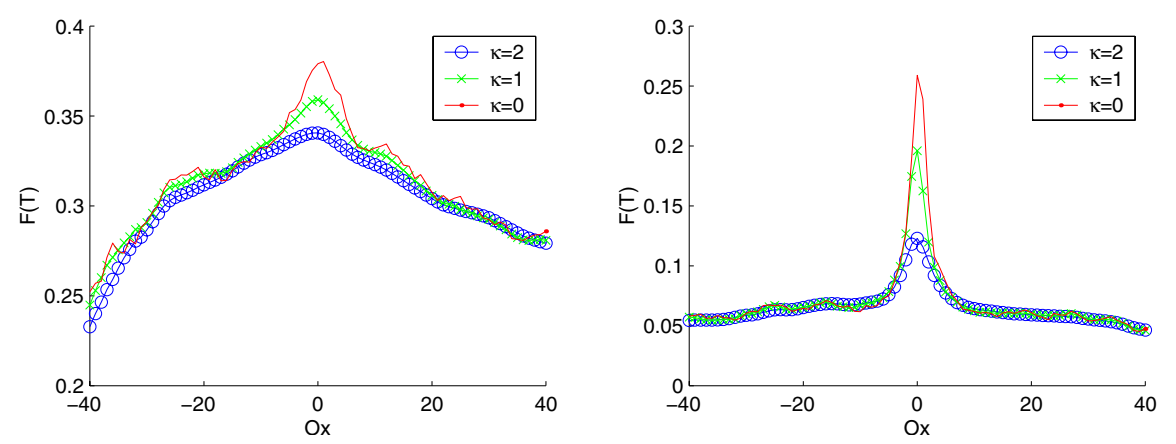

Fig. 3. Metric value for registered model translations of \pm 40 voxels $(\sim \pm 5 \mathrm{~cm})$ in the $\mathrm{x}$-axis direction for the CTA (left) and the MRA (right) data given different values of $\kappa$. The $n=1$-of-every-20 sub-sampled models and same data used in Fig. 2 were used to generate these graphs.

\subsection{Gradients of the Vascular Registration Metric}

The strength of the vascular registration system comes from the incorporation of ridge criterion into the calculation of the metric's gradient. First, to reduce the number of local maxima in the gradient space, we limit each transformed point's influence to the directions normal to its centerline. When aligned, this prevents points from traveling along a centerline and when mis-aligned, this limits, for example, horizontal vessels to inducing vertical changes in the transformation parameters. The normal plane at a point $\mathbf{x}_{i}$ is the matrix (where ${ }^{*}$ indicates co-vector transform by $\mathbf{T}$ and $\times$ indicates cross-product) 


$$
\mathbf{N}_{i}=\left(\mathbf{v}_{1 i} * \mathbf{T}\right) \times\left(\mathbf{v}_{1 i} * \mathbf{T}\right)+\left(\mathbf{v}_{2 i} * \mathbf{T}\right) \times\left(\mathbf{v}_{2 i} * \mathbf{T}\right)
$$

Therefore, the weighted, normal-plane component of the gradient is

$$
\nabla \mathbf{I}\left(\mathbf{x}_{i} \mathbf{T}\right)_{\mathbf{v}}=w_{i} \nabla \mathbf{I}_{\kappa r_{i}}\left(\mathbf{x}_{i} \mathbf{T}\right) \mathbf{N}_{i}
$$

Second, we normalize the gradient by the distribution (weighted sum) of the normal-planes' orientations. This prevents the metric gradient from being biased when the vessels models have a favored orientation. The bias matrix is

$$
\mathbf{B}=\sum_{i=1}^{n} w_{i} \mathbf{N}_{i}
$$

Therefore, the normal-plane, unbiased gradient of the metric is defined as (where $J(\mathbf{T})$ denotes the jacobian of $\mathbf{T}$ )

$$
f(\mathbf{T}) d \mathbf{T}=\frac{1}{\sum_{i=1}^{n} w_{i}} \sum_{i=1}^{n} J(\mathbf{T}) \nabla \mathbf{I}\left(\mathbf{x}_{i} \mathbf{T}\right)_{\mathbf{v}} \mathbf{B}^{-1}
$$

The utility of the proposed modifications is illustrated in Fig. 4, It depicts the components of $f(\mathbf{T}) d \mathbf{T}$ for vessels extracted from and registered with the 3D-US data. This illustrates the situation in which the vessel models match the image data exactly. There are no non-rigid effects in the data, and the ideal transformation parameter values (the identity matrix) are known. Note that the translation components are valid for a broad range of rotations, but the rotation components require the resolution of the translation components. These and similar observations are driving our research into defining a fast optimization strategy.

Using our existing gradient ascent optimization strategy, the arterial and venous phase CTA data were registered as well as the pre and post-treatment MRA data (Fig. 5). For the CTA datasets, very few vessels exist in both datasets, but the registration method is still effective. For the MRA datasets, the vessels are well registered away from the AVM but at the AVM, significant non-rigid deformations have occured since the vessels have shifted and several are missing.

In a final evaluation of the vascular registration metric, we attempted to register the arterial and venous-phase CTA data using a mutual information metric. The mutual information metric with rigid-body transform required significant intensity windowing and fine-tuning to focus its alignment on the liver. It instead favored the alignment of the bones or lungs. Even when mutual information was finally tuned to focused on the liver, surrounding organs interfered with the quality of the registration. The method presented in this paper is better for the registration of specific vascular organs.

\section{Conclusions}

The proposed vascular registration metric and its gradients are fast to calculate (require less than one-half second per evaluation), are maximal at the optimal 

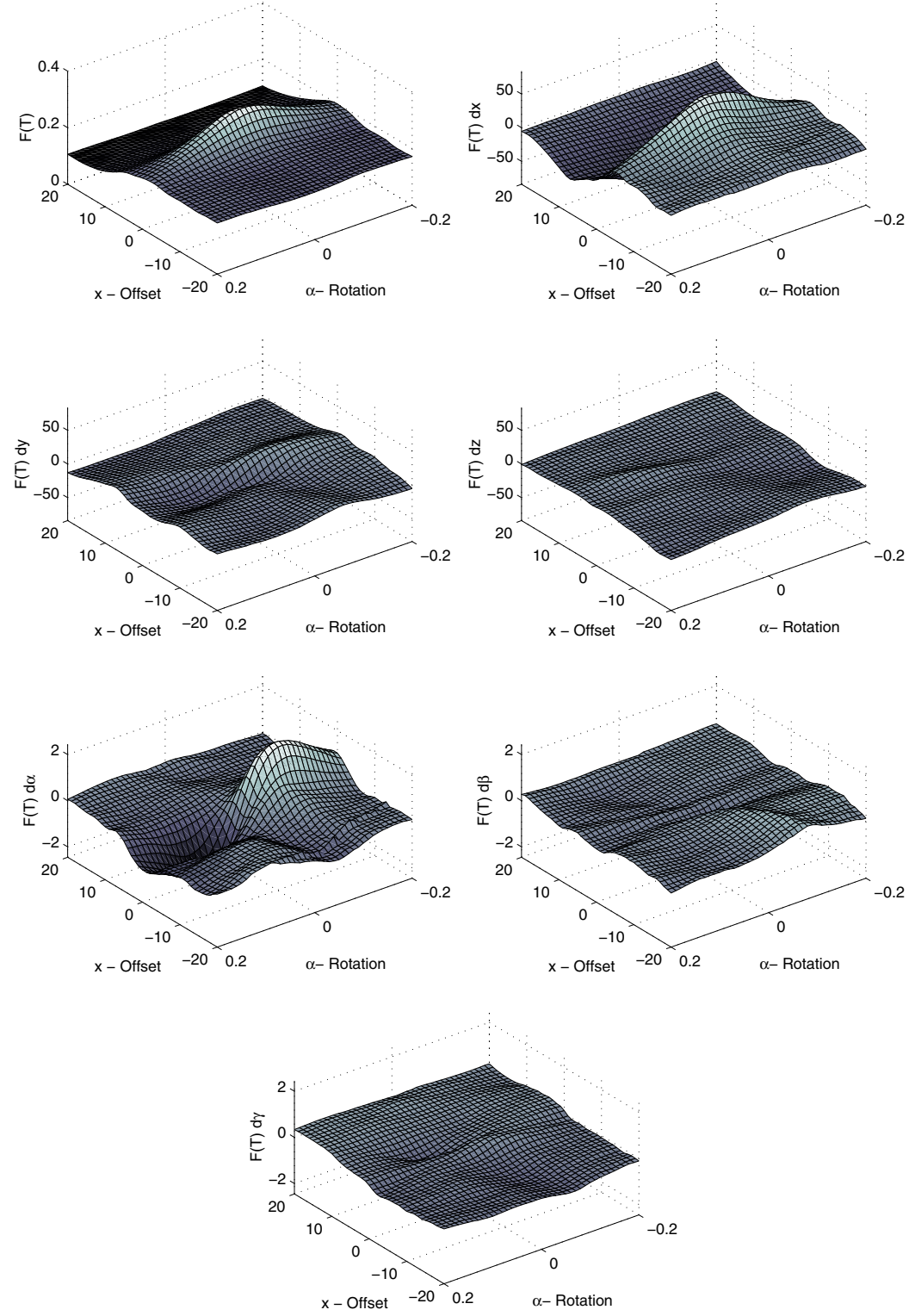

Fig. 4. Using the modified metric gradient (Equ. 6) and the 3D-US data (vessels were extracted from and registered with the same data), these graphs depict (in order from top left to bottom right) the metric and its gradient with respect to the transformation offset components $\mathbf{o}_{x}, \mathbf{o}_{y}, \mathbf{o}_{z}$ and rotation components $\alpha, \beta$, and $\gamma$ for a range of $\mathrm{x}-$ offsets ( \pm 20 voxels - y-axis on graphs) and $\alpha$ rotations ( \pm 0.2 radians - $\mathrm{x}$-axis on graphs). The metric is maximal at the ideal transformation (i.e., no transformation), the metric and its gradients vary smoothly from the ideal, gradients point towards the ideal for a large extent for the effected tranformation parameters, and gradients of un-effected transofrmation parameters remain near zero. 

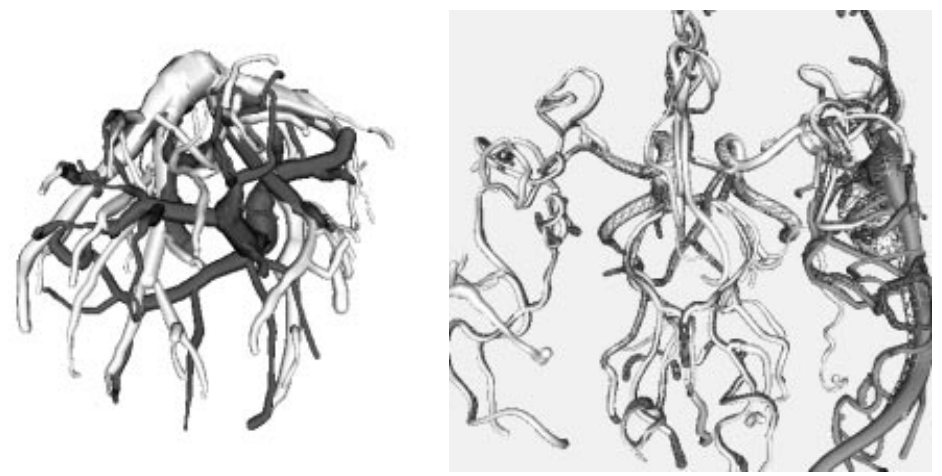

Fig. 5. (left) Gradient ascent was used to register the arterial-phase vessel models with the venous-phase CTA data. Shown are models from both sets of data (arterialphase in dark gray and venous-phase in light gray). (right) Vessel models from the registered pre and post-treatment MRA data are shown (pre-treatment in wireframe and post-treatment as surfaces).

parameter settings, and smoothly vary about that maximum. These are the features that a metric should have to be effective for image registration.

A WWW site containing additional illustrations of this and related work is at http://caddlab.rad.unc.edu. Portions of this work were implemented using the NLM's Visible Human Segmentation and Registration Toolkit ("Insight Toolkit"). This work was supported in-part by the NIH/NCI R01-CA67812, the NIH/NCI P01-A47982, and an equipment and software grant from the Microsoft Corporation. Aspects of this work have been licensed (patent pending) to Medtronic Inc. (Minn., Minn.) and R2 Technologies (Los Altos, CA).

\section{References}

1. K Harris, SN Efstraatiadis, N Maglaveras, C Pappas, J Gourassas, and G Louridas, "Model-based morphological segmentation and labeling of coronary angiograms." IEEE Transactions on Medical Imaging, 18(10):1003-1015, October 1999

2. E Bullitt, A Liu, S Aylward, C Coffey, J Stone S Mukherji, and S Pizer, "Registration of 3D Cerebral Vessels with 2D Digital Angiograms: Clinical Evaluation," Academic Radiology, 6:539-546 1999

3. N Alperin, DN Levin, and CA Pelizzari, "Retrospective registration of x-ray angiograms with MR images by using vessels as intrinsic landmarks," Journal of Magnetic Resonance Imaging, 4:139-144 1994

4. SR Aylward, E Bullitt, SM Pizer, and D Eberly, "Intensity Ridges and Widths for Tubular Object Segmentation and Registration," in IEEE Workshop on Mathematical Methods in Biomedical Image Analysis, 131-138 1996

5. PJ Yim, PL Choyke, and RM Summers, "Gray-scale skeletonization of small vessels in magnetic resonance angiography," IEEE Transactions on Medical Imaging, 19(6); 568-576, June 2000. 